\title{
The Effect of Corpus Size on Case Frame Acquisition for Discourse Analysis
}

\author{
Ryohei Sasano \\ Graduate School of Informatics, \\ Kyoto University \\ sasanoli.kyoto-u.ac.jp
}

\author{
Daisuke Kawahara \\ National Institute of Information \\ and Communications Technology \\ dk@nict.go.jp
}

\author{
Sadao Kurohashi \\ Graduate School of Informatics, \\ Kyoto University \\ kuroei.kyoto-u.ac.jp
}

\begin{abstract}
This paper reports the effect of corpus size on case frame acquisition for discourse analysis in Japanese. For this study, we collected a Japanese corpus consisting of up to 100 billion words, and constructed case frames from corpora of six different sizes. Then, we applied these case frames to syntactic and case structure analysis, and zero anaphora resolution. We obtained better results by using case frames constructed from larger corpora; the performance was not saturated even with a corpus size of 100 billion words.
\end{abstract}

\section{Introduction}

Very large corpora obtained from the Web have been successfully utilized for many natural language processing (NLP) applications, such as prepositional phrase (PP) attachment, other-anaphora resolution, spelling correction, confusable word set disambiguation and machine translation (Volk, 2001; Modjeska et al., 2003; Lapata and Keller, 2005; Atterer and Schütze, 2006; Brants et al., 2007).

Most of the previous work utilized only the surface information of the corpora, such as $n$-grams, co-occurrence counts, and simple surface syntax. This may be because these studies did not require structured knowledge, and for such studies, the size of currently available corpora is considered to have been almost enough. For instance, while Brants et al. (2007) reported that translation quality continued to improve with increasing corpus size for training language models at even size of 2 trillion tokens, the increase became small at the corpus size of larger than 30 billion tokens.

However, for more complex NLP tasks, such as case structure analysis and zero anaphora resolution, it is necessary to obtain more structured knowledge, such as semantic case frames, which describe the cases each predicate has and the types of nouns that can fill a case slot. Note that case frames offer not only the knowledge of the relationships between a predicate and its particular case slot, but also the knowledge of the relationships among a predicate and its multiple case slots. To obtain such knowledge, very large corpora seem to be necessary; however it is still unknown how much corpora would be required to obtain good coverage.

For examples, Kawahara and Kurohashi proposed a method for constructing wide-coverage case frames from large corpora (Kawahara and Kurohashi, 2006b), and a model for syntactic and case structure analysis of Japanese that based upon case frames (Kawahara and Kurohashi, 2006a). However, they did not demonstrate whether the coverage of case frames was wide enough for these tasks and how dependent the performance of the model was on the corpus size for case frame construction.

This paper aims to address these questions. We collect a very large Japanese corpus consisting of about 100 billion words, or 1.6 billion unique sentences from the Web. Subsets of the corpus are randomly selected to obtain corpora of different sizes ranging from 1.6 million to 1.6 billion sentences. We construct case frames from each corpus and apply them to syntactic and case structure analysis, and zero anaphora resolution, in order to investigate the 
relationships between the corpus size and the performance of these analyses.

\section{Related Work}

Many NLP tasks have successfully utilized very large corpora, most of which were acquired from the Web (Kilgarriff and Grefenstette, 2003). Volk (2001) proposed a method for resolving PP attachment ambiguities based upon Web data. Modjeska et al. (2003) used the Web for resolving nominal anaphora. Lapata and Keller (2005) investigated the performance of web-based models for a wide range of NLP tasks, such as MT candidate selection, article generation, and countability detection. Nakov and Hearst (2008) solved relational similarity problems using the Web as a corpus.

With respect to the effect of corpus size on NLP tasks, Banko and Brill (2001a) showed that for content sensitive spelling correction, increasing the training data size improved the accuracy. Atterer and Schütze (2006) investigated the effect of corpus size in combining supervised and unsupervised learning for two types of attachment decision; they found that the combined system only improved the performance of the parser for small training sets. Brants et al. (2007) varied the amount of language model training data from 13 million to 2 trillion tokens and applied these models to machine translation systems. They reported that translation quality continued to improve with increasing corpus size for training language models at even size of 2 trillion tokens. Suzuki and Isozaki (2008) provided evidence that the use of more unlabeled data in semisupervised learning could improve the performance of NLP tasks, such as POS tagging, syntactic chunking, and named entities recognition.

There are several methods to extract useful information from very large corpora. Search engines, such as Google and Altavista, are often used to obtain Web counts (e.g. (Nakov and Hearst, 2005; Gledson and Keane, 2008)). However, search engines are not designed for NLP research and the reported hit counts are subject to uncontrolled variations and approximations. Therefore, several researchers have collected corpora from the Web by themselves. For English, Banko and Brill (2001b) collected a corpus with 1 billion words from vari- ety of English texts. Liu and Curran (2006) created a Web corpus for English that contained 10 billion words and showed that for content-sensitive spelling correction the Web corpus results were better than using a search engine. Halacsy et al. (2004) created a corpus with 1 billion words for Hungarian from the Web by downloading 18 million pages. Others utilize publicly available corpus such as the North American News Corpus (NANC) and the Gigaword Corpus (Graff, 2003). For instance, McClosky et al. (2006) proposed a simple method of self-training a two phase parser-reranker system using NANC.

As for Japanese, Kawahara and Kurohashi (2006b) collected 23 million pages and created a corpus with approximately 20 billion words. Google released Japanese $n$-gram constructed from 20 billion Japanese sentences (Kudo and Kazawa, 2007). Several news wires are publicly available consisting of tens of million sentences. Kotonoha project is now constructing a balanced corpus of the presentday written Japanese consisting of 50 million words (Maekawa, 2006).

\section{Construction of Case Frames}

Case frames describe the cases each predicate has and what nouns can fill the case slots. In this study, case frames we construct case frames from raw corpora by using the method described in (Kawahara and Kurohashi, 2006b). This section illustrates the methodology for constructing case frames.

\subsection{Basic Method}

After parsing a large corpus by a Japanese parser $\mathrm{KNP}^{1}$, we construct case frames from modifier-head examples in the resulting parses. The problems for case frame construction are syntactic and semantic ambiguities. In other words, the resulting parses inevitably contain errors and predicate senses are intrinsically ambiguous. To cope with these problems, we construct case frames from reliable modifierhead examples.

First, we extract modifier-head examples that had no syntactic ambiguity, and assemble them by coupling a predicate and its closest case component. That is, we assemble the examples not by predicates, such as tsumu (load/accumulate), but by cou-

\footnotetext{
${ }^{1}$ http://nlp.kuee.kyoto-u.ac.jp/nl-resource/knp-e.html
} 
Table 1: Examples of Constructed Case Frames.

\begin{tabular}{|c|c|c|c|}
\hline & Case slot & Examples & Generalized examples with rate \\
\hline $\begin{array}{l}\text { tsumu }(1) \\
\text { (load) }\end{array}$ & $\begin{array}{l}g a \text { (nominative) } \\
w o \text { (accusative) } \\
n i \text { (dative) }\end{array}$ & $\begin{array}{l}\text { he, driver, friend, } \cdots \\
\text { baggage, luggage, hay, } \cdots \\
\text { car, truck, vessel, seat, } \cdots\end{array}$ & $\begin{array}{l}{[\mathrm{CT}: \text { PERSON] } 0.45,[\mathrm{NE}: \text { PERSON] }]: 0.08, \cdots} \\
{[\mathrm{CT}: \text { ARTIFACT }]: 0.31, \cdots} \\
{[\mathrm{CT}: \text { VEHICLE] }]: 0.32, \cdots}\end{array}$ \\
\hline $\begin{array}{c}\text { tsumu (2) } \\
\text { (accumulate) }\end{array}$ & $\begin{array}{l}g a \text { (nominative) } \\
w o \text { (accusative) }\end{array}$ & $\begin{array}{l}\text { player, children, party, } \cdots \\
\text { experience, knowledge, } \cdots\end{array}$ & $\begin{array}{l}\text { [CT:PERSON]:0.40, [NE:PERSON]:0.12, } \cdots \\
{[\text { CT:ABSTRACT]:0.47, } .}\end{array}$ \\
\hline$\vdots$ & $\vdots$ & & 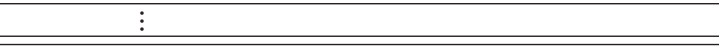 \\
\hline $\begin{array}{l}\text { hanbai (1) } \\
\text { (sell) }\end{array}$ & $\begin{array}{l}g a \text { (nominative) } \\
w o \text { (accusative) } \\
n i \text { (dative) } \\
d e \text { (locative) }\end{array}$ & $\begin{array}{l}\text { company, Microsoft, firm, } \cdots \\
\text { goods, product, ticket, } \cdots \\
\text { customer, company, user, } \cdots \\
\text { shop, bookstore, site } \cdots\end{array}$ & $\begin{array}{l}\text { [NE:ORGANIZATION]:0.16, [CT:ORGANIZATION]:0.13, } \cdots \\
{[\text { [CT:ARTIFACT]:0.40, [CT:FOOD]:0.07, } \cdots} \\
{[\text { CT:PERSON]:0.28, } \cdots} \\
{[\text { CT:FACILITY]:0.40, [CT:LOCATION]:0.39, } \cdots}\end{array}$ \\
\hline$\vdots$ & $\vdots$ & & $\vdots$ \\
\hline
\end{tabular}

ples, such as nimotsu-wo tsumu (load baggage) and keiken-wo tsumu (accumulate experience). Such couples are considered to play an important role for constituting sentence meanings. We call the assembled examples as basic case frames. In order to remove inappropriate examples, we introduce a threshold $\alpha$ and use only examples that appeared no less than $\alpha$ times in the corpora.

Then, we cluster the basic case frames to merge similar case frames. For example, since nimotsuwo tsumu (load baggage) and busshi-wo tsumu (load supplies) are similar, they are merged. The similarity is measured by using a Japanese thesaurus (The National Language Institute for Japanese Language, 2004). Table 1 shows examples of constructed case frames.

\subsection{Generalization of Examples}

When we use hand-crafted case frames, the data sparseness problem is serious; by using case frames automatically constructed from a large corpus, it was alleviated to some extent but not eliminated. For instance, there are thousands of named entities (NEs) that cannot be covered intrinsically. To deal with this problem, we generalize the examples of the case slots. Kawahara and Kurohashi also generalized examples but only for a few types. In this study, we generalize case slot examples based upon common noun categories and NE classes.

First, we generalize the examples based upon the categories that tagged by the Japanese morphological analyzer JUMAN ${ }^{2}$. In JUMAN, about 20 categories are defined and tagged to common nouns. For example, ringo (apple), inu (dog) and byoin

\footnotetext{
${ }^{2}$ http://nlp.kuee.kyoto-u.ac.jp/nl-resource/juman-e.html
}

Table 2: Definition of NE in IREX.

\begin{tabular}{ll}
\hline NE class & Examples \\
\hline ORGANIZATION & NHK Symphony Orchestra \\
PERSON & Kawasaki Kenjiro \\
LOCATION & Rome, Sinuiju \\
ARTIFACT & Nobel Prize \\
DATE & July 17, April this year \\
TIME & twelve o'clock noon \\
MONEY & sixty thousand dollars \\
PERCENT & $20 \%$, thirty percents \\
\hline
\end{tabular}

(hospital) are tagged as FOOD, ANIMAL and FACILITY, respectively. For each category, we calculate the ratio of the categorized example among all case slot examples, and add it to the case slot (e.g. [CT:FOOD]:0.07).

We also generalize the examples based upon NE classes. We use a common standard NE definition for Japanese provided by the IREX (1999). We first recognize NEs in the source corpus by using an NE recognizer (Sasano and Kurohashi, 2008); and then construct case frames from the NErecognized corpus. Similar to the categories, for each NE class, we calculate the NE ratio among all the case slot examples, and add it to the case slot (e.g. [NE:PERSON]:0.12). The generalized examples are also included in Table 1.

\section{Discourse Analysis with Case Frames}

In order to investigate the effect of corpus size on complex NLP tasks, we apply the constructed cases frames to an integrated probabilistic model for Japanese syntactic and case structure analysis (Kawahara and Kurohashi, 2006a) and a probabilistic model for Japanese zero anaphora resolution (Sasano et al., 2008). In this section, we briefly describe these models. 


\subsection{Model for Syntactic and Case Structure Analysis}

Kawahara and Kurohashi (2006a) proposed an integrated probabilistic model for Japanese syntactic and case structure analysis based upon case frames. Case structure analysis recognizes predicate argument structures. Their model gives a probability to each possible syntactic structure $T$ and case structure $L$ of the input sentence $S$, and outputs the syntactic and case structure that have the highest probability. That is to say, the system selects the syntactic structure $T_{\text {best }}$ and the case structure $L_{\text {best }}$ that maximize the probability $P(T, L \mid S)$ :

$$
\begin{aligned}
\left(T_{\text {best }}, L_{\text {best }}\right) & =\underset{(T, L)}{\operatorname{argmax}} P(T, L \mid S) \\
& =\underset{(T, L)}{\operatorname{argmax}} P(T, L, S)
\end{aligned}
$$

The last equation is derived because $P(S)$ is constant. $P(T, L, S)$ is defined as the product of a probability for generating a clause $C_{i}$ as follows:

$$
P(T, L, S)=\prod_{i=1 . . n} P\left(C_{i} \mid b_{h_{i}}\right)
$$

where $n$ is the number of clauses in $S$, and $b_{h_{i}}$ is $C_{i}$ 's modifying bunsetsu ${ }^{3}$. $P\left(C_{i} \mid b_{h_{i}}\right)$ is approximately decomposed into the product of several generative probabilities such as $P\left(A\left(s_{j}\right)=1 \mid C F_{l}, s_{j}\right)$ and $P\left(n_{j} \mid C F_{l}, s_{j}, A\left(s_{j}\right)=1\right)$, where the function $A\left(s_{j}\right)$ returns 1 if a case slot $s_{j}$ is filled with an input case component; otherwise $0 . P\left(A\left(s_{j}\right)=1 \mid C F_{l}, s_{j}\right)$ denotes the probability that the case slot $s_{j}$ is filled with an input case component, and is estimated from resultant case structure analysis of a large raw corpus. $P\left(n_{j} \mid C F_{l}, s_{j}, A\left(s_{j}\right)=1\right)$ denotes the probability of generating a content part $n_{j}$ from a filled case slot $s_{j}$ in a case frame $C F_{l}$, and is calculated by using case frames. For details see (Kawahara and Kurohashi, 2006a).

\subsection{Model for Zero Anaphora Resolution}

Anaphora resolution is one of the most important techniques for discourse analysis. In English, overt pronouns such as she and definite noun phrases such as the company are anaphors that refer to preceding entities (antecedents). On the other hand, in

\footnotetext{
${ }^{3}$ In Japanese, bunsetsu is a basic unit of dependency, consisting of one or more content words and the following zero or more function words. It corresponds to a base phrase in English.
}

Japanese, anaphors are often omitted; these omissions are called zero pronouns. Zero anaphora resolution is the integrated task of zero pronoun detection and zero pronoun resolution.

We proposed a probabilistic model for Japanese zero anaphora resolution based upon case frames (Sasano et al., 2008). This model first resolves coreference and identifies discourse entities; then gives a probability to each possible case frame $C F$ and case assignment $C A$ when target predicate $v$, input case components $I C C$ and existing discourse entities ENT are given, and outputs the case frame and case assignment that have the highest probability. That is to say, this model selects the case frame $C F_{\text {best }}$ and the case assignment $C A_{\text {best }}$ that maximize the probability $P(C F, C A \mid v, I C C, E N T)$ :

$$
\begin{aligned}
& \left(C F_{\text {best }}, C A_{\text {best }}\right) \\
& \quad=\underset{(C F, C A)}{\operatorname{argmax}} P(C F, C A \mid v, I C C, E N T)
\end{aligned}
$$

$P(C F, C A \mid v, I C C, E N T)$ is approximately decomposed into the product of several probabilities. Case frames are used for calculating $P\left(n_{j} \mid C F_{l}\right.$, $\left.s_{j}, A\left(s_{j}\right)=1\right)$, the probability of generating a content part $n_{j}$ from a case slot $s_{j}$ in a case frame $C F_{l}$, and $P\left(n_{j} \mid C F_{l}, s_{j}, A^{\prime}\left(s_{j}\right)=1\right)$, the probability of generating a content part $n_{j}$ of a zero pronoun, where the function $A^{\prime}\left(s_{j}\right)$ returns 1 if a case slot $s_{j}$ is filled with an antecedent of a zero pronoun; otherwise 0 .

$P\left(n_{j} \mid C F_{l}, s_{j}, A^{\prime}\left(s_{j}\right)=1\right)$ is similar to $P\left(n_{j} \mid C F_{l}\right.$, $\left.s_{j}, A\left(s_{j}\right)=1\right)$ and estimated from the frequencies of case slot examples in case frames. However, while $A^{\prime}\left(s_{j}\right)=1$ means $s_{j}$ is not filled with an overt argument but filled with an antecedent of zero pronoun, case frames are constructed from overt predicate argument pairs. Therefore, the content part $n_{j}$ is often not included in the case slot examples. To cope with this problem, this model also utilizes generalized examples to estimate $P\left(n_{j} \mid C F_{l}, s_{j}, A\left(s_{j}\right)=1\right)$. For details see (Sasano et al., 2008).

\section{Experiments}

\subsection{Construction of Case Frames}

In order to investigate the effect of corpus size, we constructed case frames from corpora of different sizes. We first collected Japanese sentences 
Table 4: Statistics of the Constructed Case Frames.

\begin{tabular}{c|r|r|r|r|r|r}
\hline Corpus size (sentences) & $1.6 \mathrm{M}$ & $6.3 \mathrm{M}$ & $25 \mathrm{M}$ & $100 \mathrm{M}$ & $400 \mathrm{M}$ & $1.6 \mathrm{G}$ \\
\hline \# of predicate & 2460 & 6134 & 13532 & 27226 & 42739 & 65679 \\
(type) verb & 2039 & 4895 & 10183 & 19191 & 28523 & 41732 \\
$\quad$ adjective & 154 & 326 & 617 & 1120 & 1641 & 2318 \\
$\quad$ noun with copula & 267 & 913 & 2732 & 6915 & 12575 & 21629 \\
average \# of case frames for a predicate & 15.9 & 12.2 & 13.3 & 16.1 & 20.5 & 25.3 \\
average \# of case slots for a case frame & 2.95 & 3.44 & 3.88 & 4.21 & 4.69 & 5.08 \\
average \# of examples for a case slot & 4.89 & 10.2 & 19.5 & 34.0 & 67.2 & 137.6 \\
average \# of unique examples for a case slot & 1.19 & 1.85 & 3.06 & 4.42 & 6.81 & 9.64 \\
average \# of generalized examples for a case slot & 0.14 & 0.24 & 0.37 & 0.49 & 0.67 & 0.84 \\
\hline File size(byte) & $8.9 \mathrm{M}$ & $20 \mathrm{M}$ & $56 \mathrm{M}$ & $147 \mathrm{M}$ & $369 \mathrm{M}$ & $928 \mathrm{M}$ \\
\hline
\end{tabular}

Table 3: Corpus Sizes and Thresholds.

\begin{tabular}{l|r|r|r|r|r|r}
\hline $\begin{array}{l}\text { Corpus size for case } \\
\text { frame construction } \\
\text { (sentences) }\end{array}$ & $1.6 \mathrm{M}$ & $6.3 \mathrm{M}$ & $25 \mathrm{M}$ & $100 \mathrm{M}$ & $400 \mathrm{M}$ & $1.6 \mathrm{G}$ \\
\hline $\begin{array}{l}\text { Threshold } \alpha \\
\text { introduced in Sec. 3.1 } \\
\begin{array}{l}\text { Corpus size to } \\
\text { estimate generative } \\
\text { probability (sentences) }\end{array}\end{array}$ & $1.6 \mathrm{M}$ & $3.2 \mathrm{M}$ & $6.3 \mathrm{M}$ & $13 \mathrm{M}$ & $25 \mathrm{M}$ & $50 \mathrm{M}$ \\
\hline
\end{tabular}

from the Web using the method proposed by Kawahara and Kurohashi (2006b). We acquired approximately 6 billion Japanese sentences consisting of approximately 100 billion words from 100 million Japanese web pages. After discarding duplicate sentences, which may have been extracted from mirror sites, we acquired a corpus comprising of 1.6 billion (1.6G) unique Japanese sentences consisting of approximately 25 billion words. The average number of characters and words in each sentence was $28.3,15.6$, respectively. Then we randomly selected subsets of the corpus for five different sizes; $1.6 \mathrm{M}$, $6.3 \mathrm{M}, 25 \mathrm{M}, 100 \mathrm{M}$, and $400 \mathrm{M}$ sentences to obtain corpora of different sizes.

We constructed case frames from each corpus. We employed JUMAN and KNP to parse each corpus. We changed the threshold $\alpha$ introduced in Section 3.1 depending upon the size of the corpus as shown in Table 3. Completing the case frame construction took about two weeks using 600 CPUs. Table 4 shows the statistics for the constructed case frames. The number of predicates, the average number of examples and unique examples for a case slot, and whole file size were confirmed to be heavily dependent upon the corpus size. However, the average number of case frames for a predicate and case slots for a case frame did not.

\subsection{Coverage of Constructed Case Frames}

\subsubsection{Setting}

In order to investigate the coverage of the resultant case frames, we used a syntactic relation, case structure, and anaphoric relation annotated corpus consisting of 186 web documents (979 sentences). This corpus was manually annotated using the same criteria as Kawahara et al. (2004). There were 2,390 annotated relationships between predicates and their direct (not omitted) case components and 837 zero anaphoric relations in the corpus.

We used two evaluation metrics depending upon whether the target case component was omitted or not. For the overt case component of a predicate, we judged the target component was covered by case frames if the target component itself was included in the examples for one of the corresponding case slots of the case frame. For the omitted case component, we checked not only the target component itself but also all mentions that refer to the same entity as the target component.

\subsubsection{Coverage of Case Frames}

Figure 1 shows the coverage of case frames for the overt argument, which would have tight relations with case structure analysis. The lower line shows the coverage without considering generalized examples, the middle line shows the coverage considering generalized NE examples, and the upper line shows the coverage considering all generalized examples.

Figure 2 shows the coverage of case frames for the omitted argument, which would have tight relations with zero anaphora resolution. The upper line shows the coverage considering all generalized examples, which is considered to be the upper bound of performance for the zero anaphora resolution sys- 


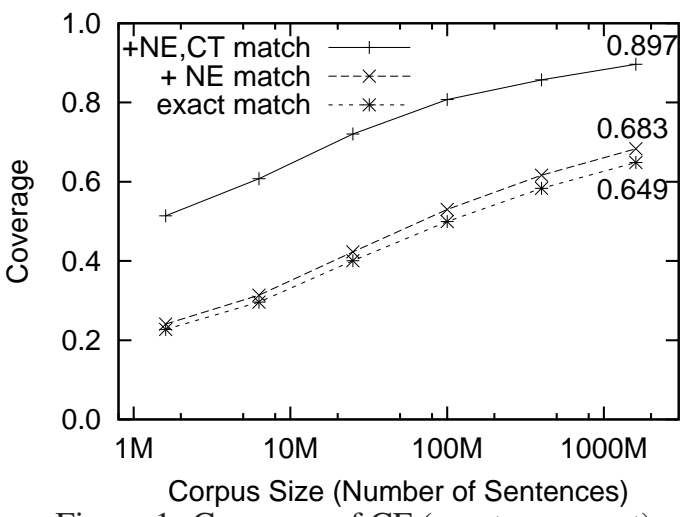

Figure 1: Coverage of CF (overt argument).

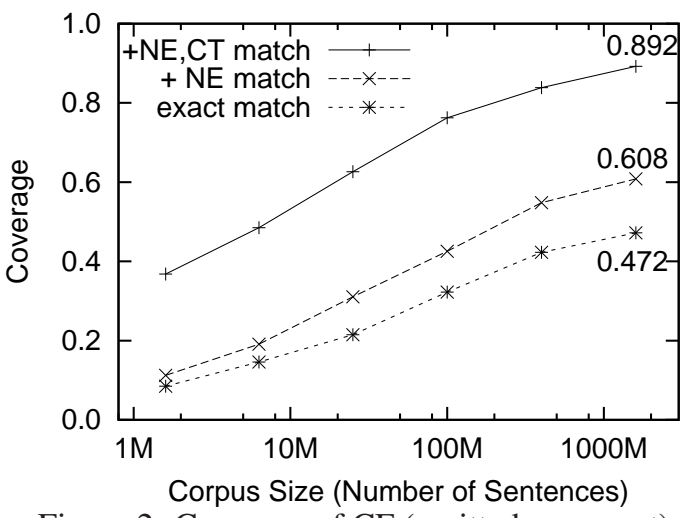

Figure 2: Coverage of CF (omitted argument).

tem described in Section 4.2. Comparing with Figure 1, we found two characteristics. First, the lower and middle lines of Figure 2 were located lower than the corresponding lines in Figure 1. This would reflect that some frequently omitted case components are not described in the case frames because the case frames were constructed from only overt predicate argument pairs. Secondly, the effect of generalized NE examples was more evident for the omitted argument reflecting the important role of NEs in zero anaphora resolution.

Both figures show that the coverage was improved by using larger corpora and there was no saturation even when the largest corpus of 1.6 billion sentences was used. When the largest corpus and all generalized examples were used, the case frames achieved a coverage of almost $90 \%$ for both the overt and omitted argument.

Figure 3 shows the coverage of case frames for each predicate type, which was calculated for both overt and omitted argument considering all generalized examples. The case frames for verbs achieved a coverage of $93.0 \%$. There were 189 predicateargument pairs that were not included case frames;

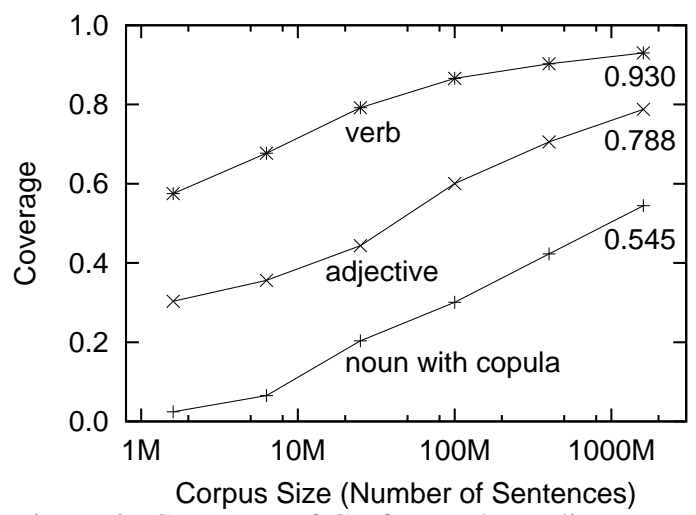

Figure 3: Coverage of CF for Each Predicate Type.

11 pairs of them were due to lack of the case frame of target predicate itself, and the others were due to lack of the corresponding example. For adjective, the coverage was $78.8 \%$. The main cause of the lower coverage would be that the predicate argument relations concerning adjectives that were used in restrictive manner, such as "oishii sushi" (delicious sushi), were not used for case frame construction, although such relations were also the target of the coverage evaluation. For noun with copula, the coverage was only $54.5 \%$. However, most predicate argument relations concerning nouns with copula were easily recognized from syntactic preference, and thus the low coverage would not quite affect the performance of discourse analysis.

\subsection{Syntactic and Case Structure Analysis}

\subsubsection{Accuracy of Syntactic Analysis}

We investigated the effect of corpus size for syntactic analysis described in Section 4.1. We used hand-annotated 759 web sentences, which was used by Kawahara and Kurohashi (2007). We evaluated the resultant syntactic structures with regard to dependency accuracy, the proportion of correct dependencies out of all dependencies ${ }^{4}$.

Figure 4 shows the accuracy of syntactic structures. We conducted these experiments with case frames constructed from corpora of different sizes. We also changed the corpus size to estimate generative probability of a case slot in Section 4.1 depending upon the size of the corpus for case frame construction as shown in Table 3. Figure 4 also in-

\footnotetext{
${ }^{4}$ Note that Kawahara and Kurohashi (2007) exclude the dependency between the last two bunsetsu, since Japanese is headfinal and thus the second last bunsetsu unambiguously depends on the last bunsetsu.
} 


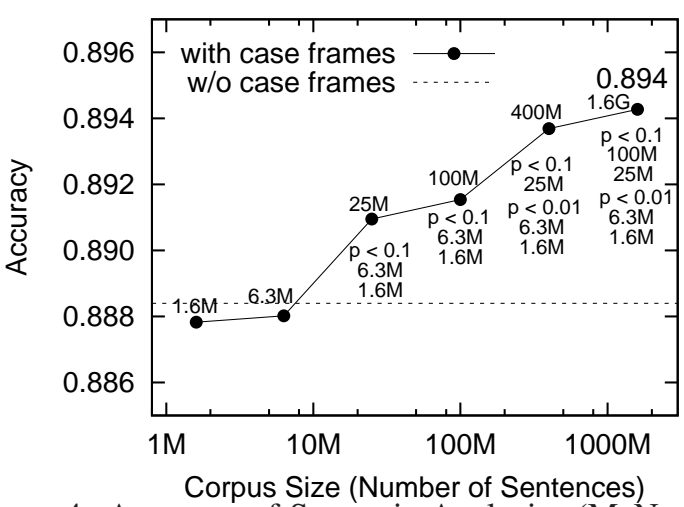

Figure 4: Accuracy of Syntactic Analysis. (McNemar's test results are also shown under each data point.)

cludes McNemar's test results. For instance, the difference between the corpus size of $1.6 \mathrm{G}$ and $100 \mathrm{M}$ sentences is significant at the $90 \%$ level $(p=0.1)$, but not significant at the $99 \%$ level $(p=0.01)$.

In Figure 4, 'w/o case frames' shows the accuracy of the rule-based syntactic parser KNP that does not use case frames. Since the model described in Section 4.1 assumes the existence of reasonable case frames, when we used case frames constructed from very small corpus, such as $1.6 \mathrm{M}$ and $6.3 \mathrm{M}$ sentences, the accuracy was lower than that of the rulebased syntactic parser. Moreover, when we tested the model described in Section 4.1 without any case frames, the accuracy was 0.885 .

We confirmed that better performance was obtained by using case frames constructed from larger corpora, and the accuracy of $0.894^{5}$ was achieved by using the case frames constructed from $1.6 \mathrm{G}$ sentences. However the effect of the corpus size was limited. This is because there are various causes of dependency error and the case frame sparseness problem is not serious for syntactic analysis.

We considered that generalized examples can benefit for the accuracy of syntactic analysis, and tried several models that utilize these examples. However, we cannot confirm any improvement.

\subsubsection{Accuracy of Case Structure Analysis}

We conducted case structure analysis on 215 web sentences in order to investigate the effect of corpus size for case structure analysis. The case markers of topic marking phrases and clausal modifiers

\footnotetext{
${ }^{5}$ It corresponds to 0.877 in Kawahara and Kurohashi's (2007) evaluation metrics.
}

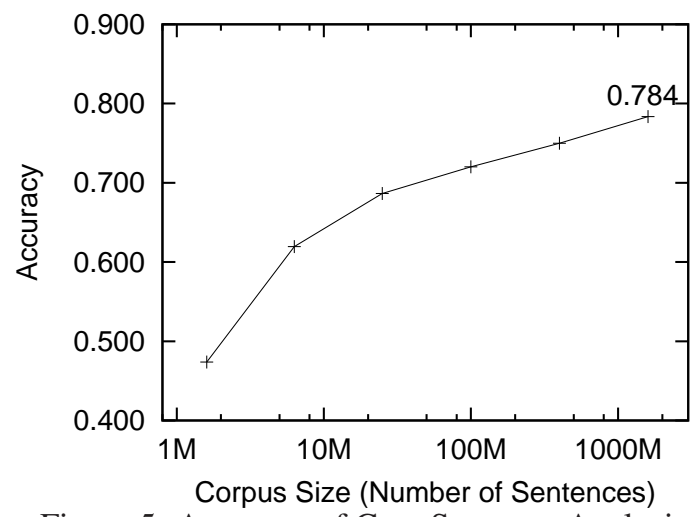

Figure 5: Accuracy of Case Structure Analysis.

Table 5: Corpus Sizes for Case Frame Construction and Time for Syntactic and Case Structure Analysis.

\begin{tabular}{l|r|r|r|r|r|r}
\hline Corpus size & $1.6 \mathrm{M}$ & $6.3 \mathrm{M}$ & $25 \mathrm{M}$ & $100 \mathrm{M}$ & $400 \mathrm{M}$ & $1.6 \mathrm{G}$ \\
\hline Time (sec.) & 850 & 1244 & 1833 & 2696 & 3783 & 5553 \\
\hline
\end{tabular}

were evaluated by comparing them with the gold standard in the corpus. Figure 5 shows the experimental results. We confirmed that the accuracy of case structure analysis strongly depends on corpus size for case frame construction.

\subsubsection{Analysis Speed}

Table 5 shows the time for analyzing syntactic and case structure of 759 web sentences. Although the time for analysis became longer by using case frames constructed from a larger corpus, the growth rate was smaller than the growth rate of the size for case frames described in Table 4.

Since there is enough increase in accuracy of case structure analysis, we can say that case frames constructed larger corpora are desirable for case structure analysis.

\subsection{Zero Anaphora Resolution}

\subsubsection{Accuracy of Zero Anaphora Resolution}

We used an anaphoric relation annotated corpus consisting of 186 web documents (979 sentences) to evaluate zero anaphora resolution. We used first 51 documents for test and used the other 135 documents for calculating several probabilities. In the 51 test documents, 233 zero anaphora relations were annotated between one of the mentions of the antecedent and corresponding predicate that had zero pronoun.

In order to concentrate on evaluation for zero anaphora resolution, we used the correct mor- 


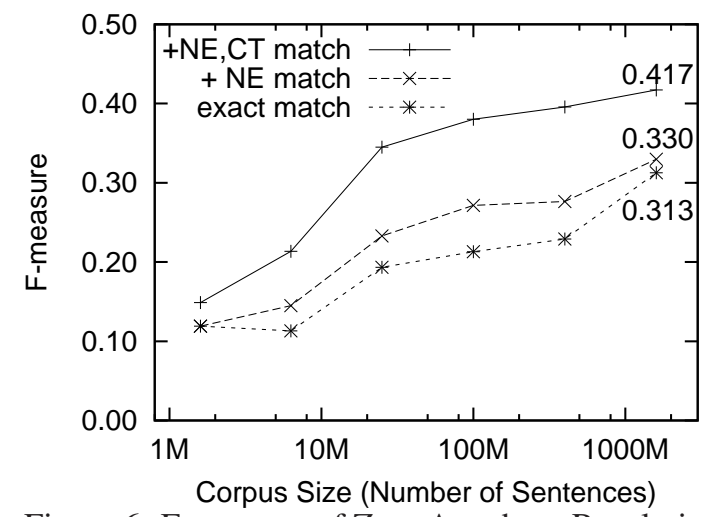

Figure 6: F-measure of Zero Anaphora Resolution.

phemes, named entities, syntactic structures and coreference relations that were manually annotated. Since correct coreference relations were given, the number of created entities was the same between the gold standard and the system output because zero anaphora resolution did not create new entities.

The experimental results are shown in Figure 6, in which F-measure was calculated by:

$$
\begin{aligned}
& R=\frac{\# \text { of correctly recognized zero anaphora }}{\# \text { of zero anaphora annotated in corpus }}, \\
& P=\frac{\# \text { of correctly recognized zero anaphora }}{\# \text { of system outputted zero anaphora }}, \\
& F=\frac{2}{1 / R+1 / P}
\end{aligned}
$$

The upper line shows the performance using all generalized examples, the middle line shows the performance using only generalized NEs, and the lower line shows the performance without using any generalized examples. While generalized categories much improved the F-measure, generalized NEs contributed little. This tendency is similar to that of coverage of case frames for omitted argument shown in Figure 2. Unlike syntactic and case structure analysis, the performance for the zero anaphora resolution is quite low when using case frames constructed from small corpora, and we can say case frames constructed from larger corpora are essential for zero anaphora resolution.

\subsubsection{Analysis Speed}

Table 6 shows the time for resolving zero anaphora in 51 web documents consisting of 278 sentences. The time for analysis became longer by using case frames constructed from larger corpora,
Table 6: Corpus Sizes for Case Frame Construction and Time for Zero Anaphora Resolution.

\begin{tabular}{l|r|r|r|r|r|r}
\hline Corpus size & $1.6 \mathrm{M}$ & $6.3 \mathrm{M}$ & $25 \mathrm{M}$ & $100 \mathrm{M}$ & $400 \mathrm{M}$ & $1.6 \mathrm{G}$ \\
\hline Time (sec.) & 538 & 545 & 835 & 1040 & 1646 & 2219 \\
\hline
\end{tabular}

which tendency is similar to the growth of the time for analyzing syntactic and case structure.

\subsection{Discussion}

Experimental results of both case structure analysis and zero anaphora resolution show the effectiveness of a larger corpus in case frame acquisition for Japanese discourse analysis. Up to the corpus size of 1.6 billion sentences, or 100 billion words, these experimental results still show a steady increase in performance. That is, we can say that the corpus size of 1.6 billion sentences is not enough to obtain case frames of sufficient coverage.

These results suggest that increasing corpus size is more essential for acquiring structured knowledge than for acquiring unstructured statistics of a corpus, such as $n$-grams, and co-occurrence counts; and for complex NLP tasks such as case structure analysis and zero anaphora resolution, the currently available corpus size is not sufficient.

Therefore, to construct more wide-coverage case frames by using a larger corpus and reveal how much corpora would be required to obtain sufficient coverage is considered as future work.

\section{Conclusion}

This paper has reported the effect of corpus size on case frame acquisition for syntactic and case structure analysis, and zero anaphora resolution in Japanese. We constructed case frames from corpora of six different sizes ranging from 1.6 million to 1.6 billion sentences; and then applied these case frames to Japanese syntactic and case structure analysis, and zero anaphora resolution. Experimental results showed better results were obtained using case frames constructed from larger corpora, and the performance showed no saturation even when the corpus size was 1.6 billion sentences.

The findings suggest that increasing corpus size is more essential for acquiring structured knowledge than for acquiring surface statistics of a corpus; and for complex NLP tasks the currently available corpus size is not sufficient. 


\section{References}

Michaela Atterer and Hinrich Schütze. 2006. The effect of corpus size in combining supervised and unsupervised training for disambiguation. In Proc. of COLING-ACL'06, pages 25-32.

Michele Banko and Eric Brill. 2001a. Mitigating the paucity-of-data problem: Exploring the effect of training corpus size on classifier performance for natural language processing. In Proc. of HLT'01.

Michele Banko and Eric Brill. 2001b. Scaling to very very large corpora for natural language disambiguation. In Proc. of ACL'01, pages 26-33.

Thorsten Brants, Ashok C. Popat, Peng Xu, Franz J. Och, and Jeffrey Dean. 2007. Large language models in machine translation. In Proc. of EMNLP-CoNLL'07, pages $858-867$.

Ann Gledson and John Keane. 2008. Using web-search results to measure word-group similarity. In Proc. of COLING'08, pages 281-288.

David Graff. 2003. English Gigaword. Technical Report LDC2003T05, Linguistic Data Consortium, Philadelphia, PA USA.

Peter Halacsy, Andras Kornai, Laszlo Nemeth, Andras Rung, Istvan Szakadat, and Vikto Tron. 2004. Creating open language resources for Hungarian. In Proc. of LREC'04, pages 203-210.

IREX Committee, editor. 1999. Proc. of the IREX Workshop.

Daisuke Kawahara and Sadao Kurohashi. 2006a. A fully-lexicalized probabilistic model for Japanese syntactic and case structure analysis. In Proc. of HLTNAACL'06, pages 176-183.

Daisuke Kawahara and Sadao Kurohashi. 2006b. Case frame compilation from the web using highperformance computing. In Proc. of LREC'06, pages 1344-1347.

Daisuke Kawahara and Sadao Kurohashi. 2007. Probabilistic coordination disambiguation in a fullylexicalized Japanese parser. In Proc. of EMNLPCoNLL'07, pages 306-314.

Daisuke Kawahara, Ryohei Sasano, and Sadao Kurohashi. 2004. Toward text understanding: Integrating relevance-tagged corpora and automatically constructed case frames. In Proc. of LREC'04, pages 1833-1836.

Adam Kilgarriff and Gregory Grefenstette. 2003. Introduction to the special issue on the web as corpus. Computational Linguistic, 29(3):333-347.

Taku Kudo and Hideto Kazawa. 2007. Web Japanese Ngram version 1, published by Gengo Shigen Kyokai.

Mirella Lapata and Frank Keller. 2005. Web-based models for natural language processing. ACM Transactions on Speech and Language Processing, 2:1:1-31.
Vinci Liu and James R. Curran. 2006. Web text corpus for natural language processing. In Proc. of EACL'O6, pages 233-240.

Kikuo Maekawa. 2006. Kotonoha, the corpus development project of the National Institute for Japanese language. In Proc. of the 13th NIJL International Symposium, pages 55-62.

David McClosky, Eugene Charniak, and Mark Johnson. 2006. Effective self-training for parsing. In Proc. of HLT-NAACL'06, pages 152-159.

Natalia N. Modjeska, Katja Markert, and Malvina Nissim. 2003. Using the web in machine learning for other-anaphora resolution. In Proc. of EMNLP-2003, pages 176-183.

Preslav Nakov and Marti Hearst. 2005. A study of using search engine page hits as a proxy for n-gram frequencies. In Proc. of RANLP'05.

Preslav Nakov and Marti A. Hearst. 2008. Solving relational similarity problems using the web as a corpus. In Proc. of ACL-HLT'08, pages 452-460.

Ryohei Sasano and Sadao Kurohashi. 2008. Japanese named entity recognition using structural natural language processing. In Proc. of IJCNLP'08, pages 607612.

Ryohei Sasano, Daisuke Kawahara, and Sadao Kurohashi. 2008. A fully-lexicalized probabilistic model for japanese zero anaphora resolution. In Proc. of COLING'08, pages 769-776.

Jun Suzuki and Hideki Isozaki. 2008. Semi-supervised sequential labeling and segmentation using giga-word scale unlabeled data. In Proceedings of ACL-HLT'O8, pages $665-673$.

The National Language Institute for Japanese Language. 2004. Bunruigoihyo. Dainippon Tosho, (In Japanese).

Martin Volk. 2001. Exploiting the WWW as a corpus to resolve PP attachment ambiguities. In Proc. of the Corpus Linguistics, pages 601-606. 